\title{
Caracterización y estructura florística de un grupo funcional vegetal del matorral espinoso tamaulipeco
}

\section{Characterization and floristic structure of a plant functional group of tamaulipan thornscrub}

\author{
Gabriel Graciano-Ávila ${ }^{1 *}$, Eduardo Alanis-Rodriguez ${ }^{1 *}$, Óscar A. Aguirre-Calderón ${ }^{1}$, Marco A. \\ González-Tagle ${ }^{1}$, Ernesto A. Rubio-Camacho ${ }^{2} \&$ J. Manuel Mata Balderas ${ }^{3}$
}

${ }^{1}$ Facultad de Ciencias Forestales, Universidad Autónoma de Nuevo León. Carretera Linares-Cd. Victoria km 145. Apartado

Postal 41. C. P. 67700, Linares, N. L. México.

${ }^{2}$ Instituto Nacional de Investigaciones Forestales, Agrícolas y Pecuarias. INIFAP, CIRPAC-C.E. Centro Altos de Jalisco.

${ }^{3}$ Gestión Estratégica y Manejo Ambiental S.C. Carretera San Miguel-Huinalá \# 935. Tercer piso. Local 38. Plaza comercial

Acanto. Apodaca, N. L. México.

*gabri.e.l@hotmail.com

\begin{abstract}
RESUMEN
La estructura, la diversidad y densidad de la vegetación son elementos importantes para la caracterización de los ecosistemas. En el presente estudio se caracterizó la estructura y diversidad de especies suculentas, arbustivas y arbóreas en un área del matorral espinoso tamaulipeco. Para ello se establecieron 70 sitios de muestreo de $10 \times 10 \mathrm{~m}\left(100 \mathrm{~m}^{2}\right)$, dentro de los cuales se realizó un censo de todas las especies arbóreas y arbustivas $\left(d_{0,10 \mathrm{~m}}>5 \mathrm{~cm}\right)$, incluyendo a las plantas suculentas. A cada individuo se le hicieron mediciones de diámetro de copa. Para cada especie se obtuvo el índice de valor de importancia (IVI), calculado a partir de tres variables, abundancia de acuerdo con el número de árboles por hectárea ( $\mathrm{n} \mathrm{ha}^{-1}$ ), dominancia a través de la cobertura del área de copa y frecuencia con base en su presencia en los sitios de muestreo. Además, se calcularon los índices diversidad de Shannon $\left(H^{\prime}\right)$ y riqueza de Margalef $\left(D_{M G}\right)$. En total se registraron 67 especies, 55 géneros y 25 familias. Este grupo funcional vegetal presenta una densidad de 3.313 ind ha-1 y una cobertura de copas de $16,671 \mathrm{~m}^{2} \mathrm{ha}^{-1}$. Acacia rigidula obtuvo el mayor valor de importancia con $8,04 \%$ de $I V I_{\text {rel }}$. La curva de rango/abundancia de especies se ajustó a una función log-normal, característica de las asociaciones vegetales maduras. Para el índice de Margalef se obtuvo un valor de $D_{M g}=8,14$, lo cual indica una alta riqueza de especies. Con lo anterior se aportan elementos cuantitativos de la vegetación arbórea, arbustiva y suculenta de un grupo funcional vegetal del matorral espinoso tamaulipeco, indicando que este tipo de vegetación presenta una alta diversidad y riqueza de especies en comparación con otras asociaciones vegetales del matorral.
\end{abstract}

Palabras clave: Plantas suculentas, densidad, índices de diversidad.

\begin{abstract}
The structure, diversity and density of vegetation are important for the characterization of ecosystems. In the present study was characterized the structure and diversity of succulent species, trees and shrubs in area of tamaulipan thornscrub of the northeast of Mexico. Was established 70 sampling sites $10 \times 10 \mathrm{~m}\left(100 \mathrm{~m}^{2}\right)$, where was perform a census of all trees and shrubs $\left(\mathrm{d}_{0.10 \mathrm{~m}}>5 \mathrm{~cm}\right)$, including succulent plants. Were made to each individual measurements of diameter of canopy. For each specie was obtained the importance value index (IVI) calculated starting of three variables, abundance according with the number of trees per hectare $\left(\mathrm{n} \mathrm{ha}^{-1}\right)$ dominance across of the coverage of the canopy area, and frequency with base in its presence in the sites of sampling. Also was calculated the Shannon index $\left(H^{\prime}\right)$ and Margalef index $\left(D_{M G}\right)$. In total were registered 67 species, 55 genres and 25 families. This plant group present a density of 3,313 ind ha ${ }^{-1}$ and the coverage of the canopy area of $16,671 \mathrm{~m}^{2} / \mathrm{ha}^{-1}$. Acacia rigidula was the one that obtained the major values of importance with $8.04 \%$ of $I V I_{r e l}$. The curve of range/abundance of species adjusted to a log-normal function, characteristic of the associations of ripe plant. For the Margalef Index was obtained a value of $D_{M G}=8.14$, this indicates a high richness of species. With this are provided quantitative elements of the arboreal vegetation, shrubby and succulent of a vegetable plant functional group of the tamaulipan thornscrub, indicating that this type of vegetation presents a high diversity and richness of species compared to other ripe plant associations of shrub.
\end{abstract}

KEYwORDS: Succulents, density, indexes of diversity. 


\section{INTRODUCCIÓN}

La vegetación de las regiones de México con clima árido y semiárido es muy variada desde el punto de vista fisonómico (Rzedowski 1978). Con 3,2\% de la superficie del territorio Nacional, el estado de Nuevo León alberga cerca del 10\% de las plantas superiores del país, con aproximadamente más de 2.400 especies (Alanís et al. 1996). La vegetación del estado ocupa 5.196.342 ha, de las cuales 348.637 ha corresponden a la superficie arbolada y las restantes 4.847.709 ha están ocupadas por vegetación de matorral y zonas áridas (SARH 1994). Cabe señalar que la vegetación de las regiones de clima seco de México se conoce como matorral xerófilo, dentro de esta denominación general se incluyen numerosos tipos de vegetación, entre los que destaca el matorral espinoso tamaulipeco y el matorral submontano (Rzedowski 2006).

El matorral espinoso tamaulipeco (MET) es una comunidad arbustiva formada por la dominancia de plantas espinosas y caducifolias. Su distribución se localiza en la porción norte de la Llanura Costera del Golfo Norte y el extremo sur de la Gran Llanura de Norteamérica con una superficie de $125.000 \mathrm{~km}^{2}$ (Foroughbakhch et al. 2005, Rzedowski 2006). Este ecosistema presenta una alta riqueza específica y diversidad de especies arbóreas y arbustivas
(Heiseke \& Foroughbakhch 1985, Alanís et al. 2008, Jiménez et al. 2013, Mora et al. 2013).

Los estudios que evalúan la estructura arbórea y arbustiva del matorral espinoso tamaulipeco del noreste de México se iniciaron desde hace décadas y se han centrado en evaluar asociaciones vegetales maduras (Reid et al. 1990, Domínguez et al. 2013, Mora et al. 2013, Ramírez-Lozano et al. 2013) o regeneradas después de diversas actividades productivas (Jiménez et al. 2012, Alanís et al. 2013, Jiménez et al. 2013). En los últimos años se han incrementado el número de investigaciones de esta temática (Alanís et al. 2015a), pero aún no se ha generado una investigación que, además de considerar el elemento arbóreo y arbustivo, cuantifique también las suculentas.

Las plantas suculentas son distribuidas ampliamente en las zonas áridas y semiáridas de México y tienen una gran importancia social, económica y ecológica (Sánchez et al. 2010, Codron et al. 2011). Por ejemplo, México es el país que alberga la mayor riqueza en especies de la familia Cactaceae, con alrededor de 850 especies que corresponden al $45 \%$ de la totalidad de esta familia nativa del continente americano. También en México se encuentra el mayor número de especies endémicas, cerca del $85 \%$ de las especies crecen solamente en este país (Hernández-Libera 1996).

\section{Área de estudio}

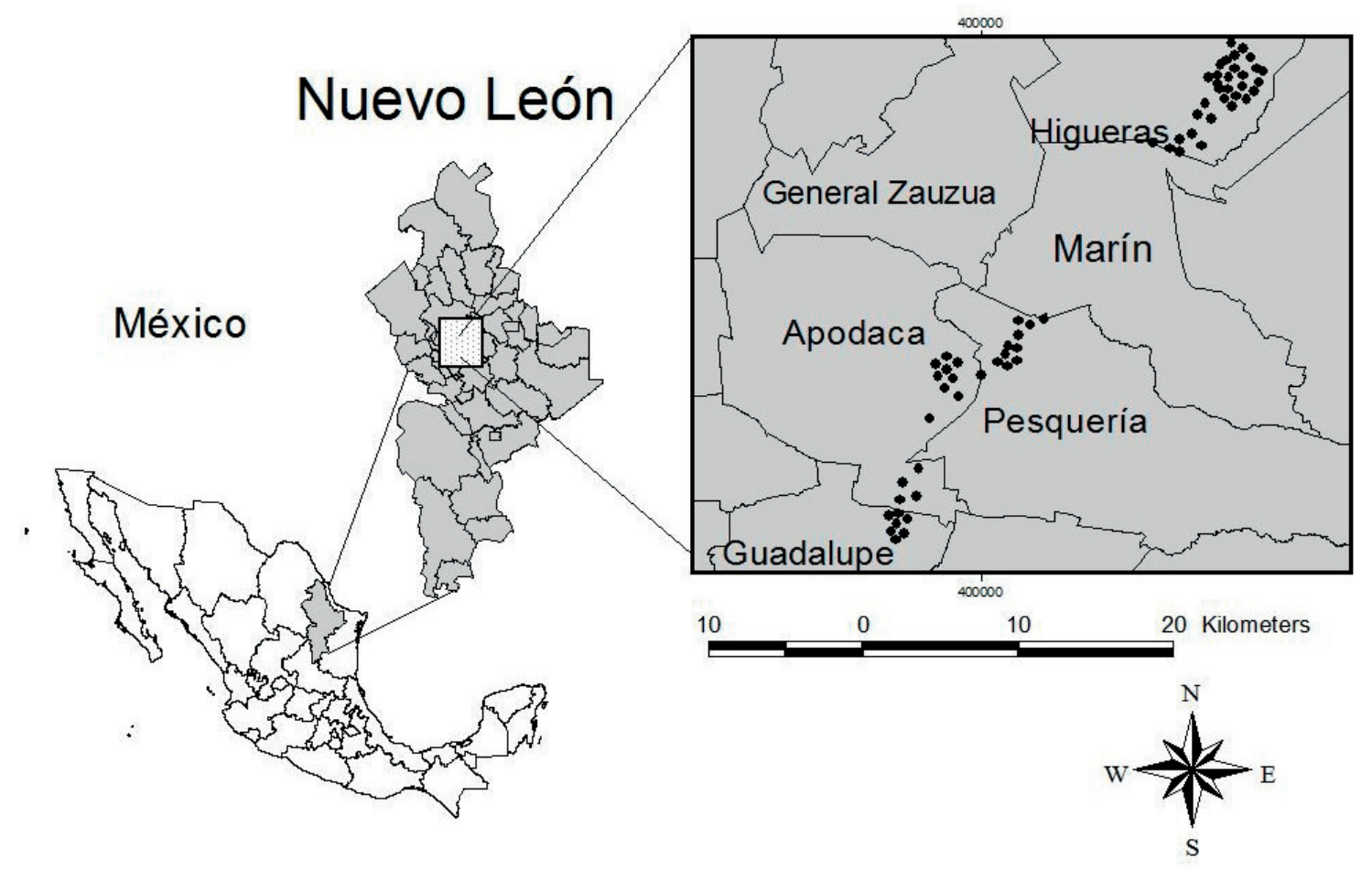

FIgURA 1. Ubicación del área de estudio. Se muestran las parcelas de muestreo. / Location of the study area. It shows the sampling plots. 
De esta forma el presente estudio tiene por objetivo generar información sobre la diversidad y composición del matorral espinoso tamaulipeco mediante la caracterización florística y estructural a nivel general, tomando como muestra especies arbóreas, arbustivas y suculentas de un grupo funcional vegetal. Esto permitirá brindar elementos para la toma de decisiones que apoyen la conservación y el manejo sustentable en este ecosistema.

\section{MATERIALES Y MÉTODOS}

Área DE ESTUdio

El estudio se llevó a cabo en una asociación vegetal de matorral espinoso tamaulipeco del noreste de México, específicamente en los municipios de Apodaca, Guadalupe, Higueras y Pesquería en el estado de Nuevo León (Fig. 1). Las coordenadas de ubicación son $25^{\circ} 47^{\prime} 07^{\prime \prime} \mathrm{N} \mathrm{y} 100^{\circ} 07^{\prime} 30^{\prime \prime}$ $\mathrm{W}$, con una amplitud altitudinal de 344 a 648 m s.n.m.

\section{TOMA DE DATOS EN CAMPO}

En la primavera de los años 2013 y 2014 se evaluó el grupo funcional vegetal arbóreo y arbustivo, así como a las plantas suculentas del área de estudio. Se establecieron aleatoriamente 70 parcelas de muestreo de $10 \times 10 \mathrm{~m}(100$ $\mathrm{m}^{2}$ ), lo cual constituye una superficie total evaluada de 7000 $\mathrm{m}^{2}$. Las parcelas que se ubicaron en áreas desprovistas de comunidad vegetal (urbanas, agrícolas, pecuarias, etc.) o presentaban una distancia menor de $100 \mathrm{~m}$ de otra parcela fueron excluidas. En cada una de ellas se realizó un censo de todas las plantas suculentas, arbustos y árboles $\geq 5 \mathrm{~cm}$ de diámetro basal $\left(d_{0,10}\right)$. A todos los individuos se les midió el diámetro de copa $\left(d_{\text {copa }}\right)$ en sentido norte-sur y este-oeste con una cinta métrica y fueron identificados por personal del laboratorio de la Facultad de Ciencias Forestales de la UANL apoyándose de la base de datos de The Plant List (2013).

\section{AnÁLISIS DE LOS DATOS}

Para cada especie se determinó su abundancia de acuerdo con el número de árboles, su dominancia en función del área de copa y su frecuencia con base en su presencia en los sitios de muestreo. Las variables relativizadas se utilizaron para obtener un valor ponderado a nivel de especie denominado Índice de Valor de Importancia (IVI), que adquiere valores porcentuales en una escala de 0 a 100 (Müeller-Dombois \& Ellenberg 1974). Las fórmulas para estos cálculos se describen a continuación.

$$
\begin{gathered}
A_{i}=N_{i} / E \\
A R_{i}=\left(A_{i} \sum_{i=1 \ldots n} A_{i}\right) \times 100
\end{gathered}
$$

donde $A_{i}$ es la abundancia absoluta, $A R_{i}$ es la abundancia relativa de la especie $i$ respecto a la abundancia total, $N_{i}$ es el número de individuos de la especie $i$, y $E$ la superficie de muestreo (ha).

La cobertura relativa se evaluó mediante:

$$
\begin{aligned}
& D_{i}=A b_{i} / E(h a) \\
& D R_{i}=\left(D_{i} / \sum_{i=1 . \ldots n} D_{i}\right) \times 100
\end{aligned}
$$

donde $D_{i}$ es la cobertura absoluta, $D R_{i}$ es cobertura relativa de la especie $i$ respecto a la cobertura, $A b$ el área de copa de la especie $i$ y $E$ la superficie (ha).

$\mathrm{La}$ frecuencia relativa se obtuvo con la siguiente ecuación:

$$
\begin{gathered}
F_{i}=P_{i} / N S \\
F R_{i}=\left(F_{i} / \sum_{i=1 \ldots n} F_{i}\right) \times 100
\end{gathered}
$$

donde $F_{i}$ es la frecuencia absoluta, $F R_{i}$ es la frecuencia relativa de la especie $i$ respecto a la suma de las frecuencias, $P_{i}$ es el número de sitios en el que está presente la especie $i$ y $N S$ el número total de sitios de muestreo.

El índice de valor de importancia (IVI) se define como (Müeller-Dombois \& Ellenberg 1974):

$$
I V I=\frac{\sum_{n}^{i=1}\left(A R_{i}, D R_{i}, F R_{i}\right)}{3}
$$

El Índice de Valor de Importancia Familiar (IVIF) adquiere valores de 0 a $100 \%$ y se calculó de la siguiente manera (Whittaker 1972, Moreno 2001):

$$
I V I F=\frac{\sum_{n}^{i=1}\left(A R F_{i}, D R F_{i}, F R F_{i}\right)}{3}
$$

donde $A R F_{i}=$ abundancia relativa de la familia $i$ respecto a la abundancia total, $D R F_{i}=$ dominancia relativa de la familia $i$ respecto a la dominancia total, y $F R F_{i}=$ frecuencia relativa de la familia $i$ respecto a la frecuencia total.

Para describir la estructura del grupo funcional vegetal en términos de la abundancia de cada especie se utilizaron curvas de rango/abundancia (rango de especies/abundancia de especies) (Magurran 2004), las cuales muestran la relación entre el valor de abundancia absoluta de las especies en función de un arreglo secuencial que va de la más común a la más rara (Martella et al. 2012). En la actualidad existen muchos modelos que se utilizan para describir la diversidad de especies en una asociación vegetal. Sin embargo, en este estudio solo se analizan tres de los más conocidos: el 
modelo Poison de la serie normal logarítmica, el modelo Serie Geométrica y el Modelo Neutral de Alonso y Mackane (Prado et al. 2014). Se ajustaron los modelos mediante el método de máxima verosimilitud. Los programas utilizados para dicho ajuste son dos principalmente, el $\mathrm{R}$ v 3.1.2 (R Development Core Team 2011) y el RStudio v 0,99 ("RStudio," n.d.), utilizando las rutinas de Prado et al. (2014). La selección de los modelos se llevó a cabo mediante dos métodos, visual y estadístico. El método visual evalúa el comportamiento de los datos predichos contra los observados de manera gráfica, esto es, se observa que la distribución de los datos sea similar. El método estadístico utilizado fue el criterio de información de Akaike (AIC), en el que se comparan los modelos seleccionados tomando en cuenta su ajuste y su complejidad, cuando se comparan dos modelos utilizando este método la selección del mejor modelo está en función del menor valor en AIC. Sin embargo, también se toma en cuenta el valor delta AIC (dAIC), que compara los modelos con base a su resultado de AIC, menos el mínimo AIC. Cuando el dAIC es menor a 2 quiere decir que no hay diferencia entre los modelos.

Para estimar la diversidad alfa se utilizó el índice de Margalef $\left(D_{M g}\right)$ y el índice de Shannon-Weaver $\left(H^{\prime}\right)$ mediante las ecuaciones (Shannon 1948, Magurran 2004):

$$
\begin{gathered}
D_{M g}=\frac{(S-1)}{\ln (N)} \\
H^{\prime}=-\sum_{i=1}^{S} p_{i} \times \ln \left(p_{i}\right) \\
p_{i}=n_{i} / N
\end{gathered}
$$

donde $S$ es el número de especies presentes, $N$ es el número total de individuos, $n_{i}$ es el número de individuos de la especie $i$ y $p_{i}$ es la proporción de individuos de la especie $i$ respecto al total de individuos (es decir la abundancia relativa de la especie $i$ ).

\section{RESULTADOS}

\section{RiQueZA}

Se registraron 47 especies arbóreas y arbustivas y 20 especies suculentas. Las especies arbóreas y arbustivas están distribuidas en 41 géneros y 22 familias (Tabla 1), las familias con mayor número de especies fueron, Fabaceae y Rhamnaceae con 10 y 5 especies, respectivamente, seguidas de las familias Euphorbiaceae y Verbenaceae con cuatro especies cada una. Estas cuatro familias incluyeron 18 géneros y 23 especies, lo que constituye $34,3 \%$ de la vegetación registrada en las 70 parcelas de muestreo.

Las 20 especies suculentas se encuentran distribuidas en 14 géneros y 3 familias (Tabla 1). La familia Cactaceae presentó el mayor número de especies con 17 , seguida de las familias Asparagaceae con dos especies y Bromeliaceae con una especie, lo que constituye el 29,8\% de la vegetación registrada en el grupo funcional vegetal evaluado.

\section{ESTRUCTURA}

El grupo funcional evaluado presentó una densidad de 3.313 ind $\mathrm{ha}^{-1}$. Las familias del grupo de las arbóreas y arbustivas con mayor densidad fueron Fabaceae (16,93\%), Asteraceae $(15,85 \%)$ y Verbenaceae $(14,28)$, sumando un $47,06 \%$ del total. La cobertura de copas fue de $16,671 \mathrm{~m}^{2} \mathrm{ha}^{-1}$, lo que indica que hay una cobertura del $100 \%$ y sobreposición de copas. Las familias que presentaron mayor cobertura fueron Fabaceae $(27,26 \%)$ y Rutaceae $(21,77 \%)$, sumando un $49,03 \%$ del total. Las familias Fabaceae, Rutaceae, Asteraceae, Scrophulariaceae y Verbenaceae fueron las más importantes de acuerdo con los valores del Índice de Valor de Importancia Familiar (IVIF), sumando 55,3\% del total del grupo funcional estudiado. Las familias Passifloraceae, Krameriaceae, Solanaceae, Celastraceae, Rubiaceae, Caesalpiniaceae fueron las que presentaron menos de $1 \%$ de IVIF (Tabla 2).

En relación al grupo de las suculentas, las familias con mayor densidad fueron Cactaceae (4,71\%) y Asparagaceae $(4,01 \%)$ sumando $8,72 \%$ del total. La mayor cobertura la presentó la familia Asparagaceae con 3,75\% del total. Las familias con mayor peso ecológico fueron Cactaceae $(5,34 \%)$ y Asparagaceae $(4,7 \%)$, sumando $10 \%$ del total del grupo funcional evaluado (Tabla 2).

Las especies con mayor densidad fueron Lippia graveolens (13,9\%), Chromolaena odorata (9,48\%), Acacia rigidula (8,03\%) y Leucophyllum frutescens (7,33\%) sumando 38,8\% del total. Las especies con mayor cobertura fueron Helietta parvifolia (13,07\%), Acacia rigidula (10,5\%), Zanthoxylum fagara $(8,7 \%)$ y Cordia boissieri (8,4\%), sumando un $40,7 \%$ del total. Las especies que se encuentran presentes en el mayor número de sitios fueron Leucophyllum frutescens en 56 sitios (80\%), Acacia rigidula en 45 sitios $(64,3 \%)$ y Eysenhardtia texana en 43 sitios $(61,4 \%)$. Según los valores del Índice de Valor de Importancia (IVI) Acacia rigidula (8,04\%), Leucophyllum frutescens (7,19\%), Helietta parvifolia $(6,71)$ y Lippia graveolens $(6,43)$ fueron las más sobresalientes, al registrar los valores más altos, sumando $28,38 \%$ del total del grupo funcional vegetal evaluado. El 58,2\% de las especies (39 de 67) presentan valores de IVI inferiores de 1,0\% (Tabla 3 ).

En relación al grupo de las suculentas, las especies con mayor densidad fueron Agave lechuguilla $(2,29 \%)$ y Agave aspérrima $(1,21 \%)$, sumando $3,5 \%$ del total. La mayor cobertura la presentó la especie Agave lechuguilla $(0,42 \%)$ y Hechtia glomerata $(0,14 \%)$ sumando $0,56 \%$ del total. Las especies con mayor peso ecológico fueron Agave lechuguilla $(1,86 \%)$ y Agave asp $\neq$ rrima $(1,17 \%)$ (Tabla 3 ). 
Gayana Bot. 75(1), 2018

TABLA 1. Nombre científico, nombre común, familia y forma de crecimiento de las especies registradas en el área de estudio (ordenadas alfabéticamente por nombre científico). / Scientific name, common name, family and growth form of the species recorded in the study area (listed alphabetically by scientific name).

\begin{tabular}{|c|c|c|c|}
\hline Nombre CIENTíFICO & NomBre COMÚN & FAMILIA & $\begin{array}{l}\text { FORMA DE } \\
\text { CRECIMIENTO }\end{array}$ \\
\hline Acacia farnesiana (L.) Willd. & Huizache & Fabaceae & Arbustiva \\
\hline Acacia greggii A. Gray & Uña de gato & Fabaceae & Arbustiva \\
\hline Acacia rigidula Benth. & Gavia & Fabaceae & Arbustiva \\
\hline Acacia wrightii A. Gray & Uña de gato & Fabaceae & Arbustiva \\
\hline Agave asperrima Jacobi & Agave cenizo & Asparagaceae & Suculenta \\
\hline Agave lechuguilla Torr. & Lechuguilla & Asparagaceae & Suculenta \\
\hline Aloysia gratissima (Gillies \& Hook.) Tronc. & Aloysia & Verbenaceae & Arbustiva \\
\hline $\begin{array}{l}\text { Ariocarpus retusus Scheidw. subsp. trigonus (F.A.C. Weber) } \\
\text { E.F. Anderson \& Fitz Maurice }\end{array}$ & Chaute & Cactaceae & Suculenta \\
\hline Bernardia myricifolia (Scheele) S. Watson. & Oreja de ratón & Euphorbiaceae & Arbustiva \\
\hline Castela tortuosa Liebm. & Chaparro amargoso & Simaroubaceae & Arbustiva \\
\hline Celtis pallida Torr. & Granjeno & Cannabaceae & Arbustiva \\
\hline Cercidium macrum I.M. Johnst. & Palo verde & Fabaceae & Arbórea \\
\hline Chromolaena odorata (L.) R.M. King y H. Rob. & Ageratina & Asteraceae & Arbustiva \\
\hline Colubrina greggii S. Watson. & Manzanita & Rhamnaceae & Arbórea \\
\hline Colubrina texensis (Torr. \& A. Gray) A. Gray & Manzanita de los puercos & Rhamnaceae & Arbustiva \\
\hline Condalia hookeri M.C. Johnst. & Brasil & Rhamnaceae & Arbórea \\
\hline Cordia boissieri A. DC. & Anacahuita & Boraginaceae & Arbustiva \\
\hline Corynopuntia schottii (Engelm.) F.M. Knuth & Perritos & Cactaceae & Suculenta \\
\hline Coryphantha nickelsiae (K.Brandegee) Britton \& Rose & Biznaguita partida & Cactaceae & Suculenta \\
\hline Coryphantha salinensis A. Zimmerman ex Dicht \& A. Luethy & Biznaguita partida mayor & Cactaceae & Suculenta \\
\hline Croton torreyanus Müll. Arg. & Croto & Euphorbiaceae & Arbustiva \\
\hline Cylindropuntia leptocaulis (DC.) F.M. Knuth & Tasajillo & Cactaceae & Suculenta \\
\hline Diospyros texana Scheele & Chapote prieto & Ebenaceae & Arbórea \\
\hline Dodonaea viscosa (L.) Jacq. & Dodonea & Sapindaceae & Arbustiva \\
\hline Ebenopsis ebano (Berland.) Barneby \& J.W. Grimes & Ébano & Fabaceae & Arbórea \\
\hline Echinocactus horizonthalonius Lem. & Mancacaballo & Cactaceae & Suculenta \\
\hline Echinocactus texensis Hopffer & Mancacaballo anguloso & Cactaceae & Suculenta \\
\hline Echinocereus parkeri N.P. Taylor & Pitayo & Cactaceae & Suculenta \\
\hline Echinocereus pentalophus (DC.) Lem. & Pitayo postrado & Cactaceae & Suculenta \\
\hline Echinocereus poselgeri Lem. & Sacazil & Cactaceae & Suculenta \\
\hline Ehretia anacua (Terán \& Berland.) I.M. Johnst. & Anacua & Boraginaceae & Arbórea \\
\hline Eysenhardtia texana Scheele & Vara dulce & Fabaceae & Arbustiva \\
\hline Ferocactus hamatacanthus (Muehlenpf.) Britton \& Rose & Biznaga ganchuda & Cactaceae & Suculenta \\
\hline Forestiera angustifolia Torr. & Panalero & Oleaceae & Arbustiva \\
\hline Gymnosperma glutinosum (Spreng.) Less. & Pegajosilla & Asteraceae & Arbustiva \\
\hline Havardia pallens (Benth.) Britton \& Rose & Tenaza & Fabaceae & Arbórea \\
\hline Hechtia glomerata Zucc. & Guapilla & Bromeliaceae & Suculenta \\
\hline
\end{tabular}




\begin{tabular}{|c|c|c|c|}
\hline NombRe CIENTíFICO & NOMBRE COMÚN & FAMILIA & $\begin{array}{c}\text { FORMA DE } \\
\text { CRECIMIENTO }\end{array}$ \\
\hline Helietta parvifolia (A. Gray) Benth. & Barreta & Rutaceae & Arbustiva \\
\hline Jatropha dioica Sessé & Sangre de drago & Euphorbiaceae & arbustiva \\
\hline Krameria ramosissima (A. Gray) S. Watson & Krameria & Krameriaceae & arbustiva \\
\hline Lantana achyranthifolia Desf. & Lantanilla & Verbenaceae & Arbustiva \\
\hline Lantana camara $\mathrm{L}$. & Lantana & Verbenaceae & Arbustiva \\
\hline Leucaena pulverulenta (Schltdl.) Benth. & Leucaena & Fabaceae & Arbórea \\
\hline Leucophyllum frutescens (Berland.) I.M. Johnst. & Cenizo & Scrophulariaceae & Arbustiva \\
\hline Lippia graveolens Kunth & Oreganillo & Verbenaceae & Arbustiva \\
\hline Lycium berlandieri Dunal & Cilindrillo & Solanaceae & Arbustiva \\
\hline Mammillaria heyderi Muehlenpf. & Biznaga de chilitos & Cactaceae & Suculenta \\
\hline Mammillaria prolifera (Mill.) Haw. & Biznaguita & Cactaceae & Suculenta \\
\hline Neomammillaria candida (Scheidw.) Britton \& Rose & Bola de nieve & Cactaceae & Suculenta \\
\hline Neopringlea integrifolia (Hemsley) S. Watson & Corvagallina & Salicaceae & arbórea \\
\hline Nicotiana glauca Graham & Tabaquillo & Solanaceae & arbustiva \\
\hline Opuntia engelmannii (Salm-Dyck) ex Engelm. & Nopal & Cactaceae & Suculenta \\
\hline Parkinsonia acuelata L. & Retama & Caesalpiniaceae & Arbórea \\
\hline Parthenium incanum Kunth. & Mariola & Asteraceae & Arbustiva \\
\hline Porlieria angustifolia (Engelm.) A. Gray & Guayacán & Zygophyllaceae & Arbustiva \\
\hline Prosopis glandulosa Torr. & Mezquite & Fabaceae & Arbórea \\
\hline Randia laetevirens Standl. & Crucillo & Rubiaceae & Arbórea \\
\hline Rhamnus humboldtiana Willd. ex Schut. & Coyotillo & Rhamnaceae & arbustiva \\
\hline Ricinus communis L. & Higuerilla & Euphorbiaceae & Arbustiva \\
\hline Schaefferia cuneifolia A. Gray & Capul & Celastraceae & Arbustiva \\
\hline Sclerocactus scheeri (Salm-Dyck) N.P. Taylor & Biznaguita ganchuda & Cactaceae & Suculenta \\
\hline Sideroxylon celastrinum (Kunth) T.D. Penn. & Coma & Sapotaceae & Arbustiva \\
\hline Turbinicarpus saueri (Boed.) John \& Říha & Biznaguita & Cactaceae & Suculenta \\
\hline Turnera diffusa Willd. ex Schult. & Damiana & Passifloraceae & Arbustiva \\
\hline Yucca filifera Chabaud & Yuca & Asparagaceae & Arbórea \\
\hline Zanthoxylum fagara (L.) Sarg. & Colima & Rutaceae & Arbustiva \\
\hline Ziziphus obtusifolia (Hook. ex Torr. \& A. Gray) A. Gray & Clepe & Rhamnaceae & Arbustiva \\
\hline
\end{tabular}

CURVA DE RANGO/ABUNDANCIA DE LAS ESPECIES

En la Figura 2 se muestran las 3 curvas de rango/abundancia para todas las especies registradas en el muestreo de la vegetación. Solo la primera curva se ajusta gráficamente a la distribución Poisson-Lognormal, las otras 2 curvas, Geométrica y la distribución Neutral de Alonso y Mackane, no se ajustan a la distribución de los datos (Fig. 2, Tabla 4).
ÍNDICES DE DIVERSIDAD DE ESPECIES

La riqueza específica del grupo funcional vegetal del matorral espinoso tamaulipeco estudiado fue de 67 especies y presentó un índice de Margalef de $D_{M g}=8,14$. En relación al valor de diversidad de especies, el valor del índice de Shannon fue de $H^{\prime}=3,36$. 

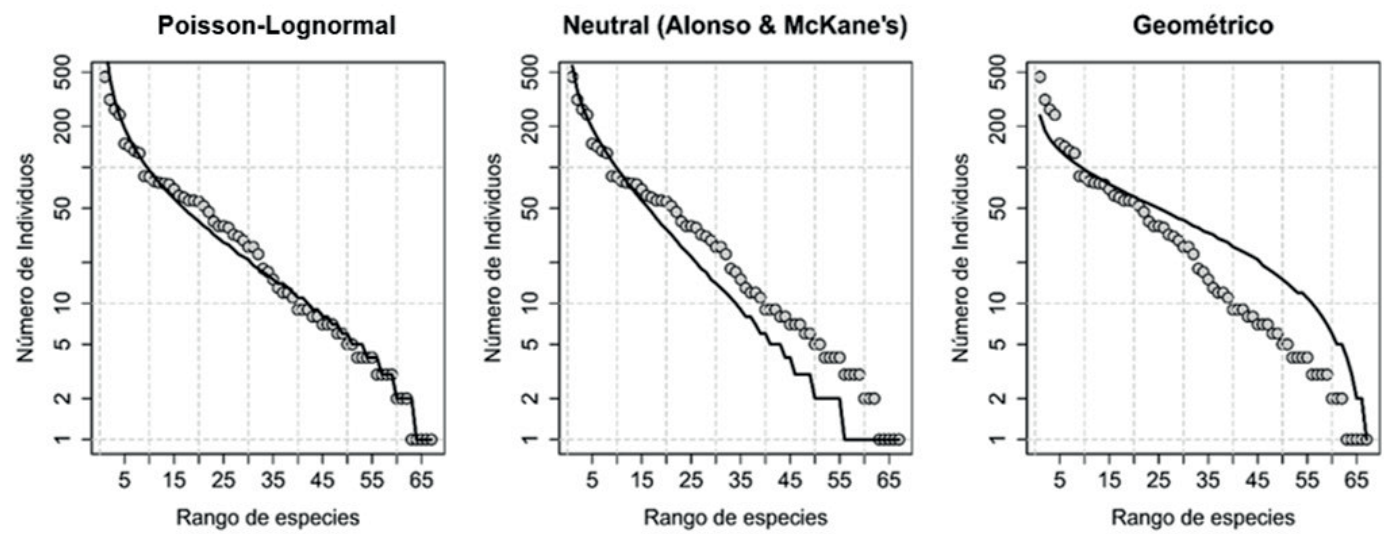

FiguRA 2. Curvas observadas de rango/abundancia de las especies para el matorral espinoso tamaulipeco del área de estudio, en donde las especies se agrupan de la más común a la más rara. / Curves observed range/abundance of species for the tamaulipan thornscrub of the study area, wherein species are grouped of the common to the rare.

TABLA 2. Parámetros estructurales estimados para las familias registradas en el área de estudio. IVIF = Índice de Valor de Importancia Familiar. Las familias están ordenadas en forma descendente según su IVIF. / Structural parameters estimated for the families registered in the study area. IVIF = Index of Value Family Importance. Families are sorted in descending order according to their IVIF.

\begin{tabular}{|c|c|c|c|c|c|c|c|}
\hline \multirow{3}{*}{ FAMILIA } & \multicolumn{2}{|c|}{ ABUNDANCIA } & \multicolumn{2}{|c|}{ DOMINANCIA } & \multicolumn{2}{|c|}{ FRECUENCIA } & \multirow{3}{*}{ IVIF } \\
\hline & ABsoluta & RELATIVA & Absoluta & RELATIVA & ABsoluta & RELATIVA & \\
\hline & $\left(\mathrm{n} \mathrm{ha}^{-1}\right)$ & $(\%)$ & $\left(\mathrm{m}^{2} \mathrm{ha}^{-1}\right)$ & $(\%)$ & & $(\%)$ & \\
\hline Fabaceae & 561 & 16,93 & 4544,93 & 27,26 & 235,71 & 20,55 & 21,58 \\
\hline Rutaceae & 259 & 7,82 & 3628,55 & 21,77 & 81,43 & 7,10 & 12,23 \\
\hline Asteraceae & 525 & 15,85 & 875,69 & 5,25 & 20,00 & 1,74 & 7,61 \\
\hline Scrophulariaceae & 243 & 7,33 & 1211,61 & 7,27 & 80,00 & 6,97 & 7,19 \\
\hline Verbenaceae & 473 & 14,28 & 247,49 & 1,48 & 50,00 & 4,36 & 6,71 \\
\hline Boraginaceae & 87 & 2,63 & 1405,72 & 8,43 & 58,57 & 5,11 & 5,39 \\
\hline Cactaceae & 156 & 4,71 & 16,44 & 0,10 & 128,57 & 11,21 & 5,34 \\
\hline Rhamnaceae & 238 & 7,18 & 266,92 & 1,60 & 77,14 & 6,72 & 5,17 \\
\hline Asparagaceae & 133 & 4,01 & 624,73 & 3,75 & 72,86 & 6,35 & 4,70 \\
\hline Cannabaceae & 60 & 1,81 & 966,66 & 5,80 & 45,71 & 3,99 & 3,86 \\
\hline Ebenaceae & 36 & 1,09 & 824,56 & 4,95 & 27,14 & 2,37 & 2,80 \\
\hline Euphorbiaceae & 116 & 3,50 & 133,89 & 0,80 & 45,71 & 3,99 & 2,76 \\
\hline Oleaceae & 52 & 1,57 & 483,08 & 2,90 & 34,29 & 2,99 & 2,49 \\
\hline Sapotaceae & 37 & 1,12 & 445,37 & 2,67 & 34,29 & 2,99 & 2,26 \\
\hline Salicaceae & 37 & 1,12 & 379,22 & 2,27 & 27,14 & 2,37 & 1,92 \\
\hline Zygophyllaceae & 69 & 2,08 & 54,20 & 0,33 & 32,86 & 2,86 & 1,76 \\
\hline Sapindaceae & 57 & 1,72 & 267,76 & 1,61 & 18,57 & 1,62 & 1,65 \\
\hline Simaroubaceae & 23 & 0,69 & 166,16 & 1,00 & 30,00 & 2,62 & 1,44 \\
\hline Bromeliaceae & 32 & 0,97 & 23,74 & 0,14 & 22,86 & 1,99 & 1,03 \\
\hline Passifloraceae & 56 & 1,69 & 13,29 & 0,08 & 4,29 & 0,37 & 0,71 \\
\hline Krameriaceae & 47 & 1,42 & 23,29 & 0,14 & 2,86 & 0,25 & 0,60 \\
\hline Solanaceae & 8 & 0,24 & 44,43 & 0,27 & 8,57 & 0,75 & 0,42 \\
\hline Celastraceae & 5 & 0,15 & 6,54 & 0,04 & 4,29 & 0,37 & 0,19 \\
\hline Rubiaceae & 2 & 0,06 & 3,07 & 0,02 & 2,86 & 0,25 & 0,11 \\
\hline \multirow[t]{2}{*}{ Caesalpiniaceae } & 1 & 0,03 & 13,74 & 0,08 & 1,43 & 0,12 & 0,08 \\
\hline & 3313 & 100 & 16671,06 & 100 & 1147,14 & 100 & 100 \\
\hline
\end{tabular}


Caracterización del matorral espinoso tamaulipeco: Graciano-ÁviLA, G. ET AL.

TABLA 3, Parámetros estructurales estimados para las especies registradas en el área de estudio, IVI = Índice de Valor de Importancia, Las especies están ordenadas en forma descendente según su IVI, / Structural parameter estimates for the species recorded in the study area, IVI $=$ Index of Importance Value, The species are sorted in descending order by IVI,

\begin{tabular}{|c|c|c|c|c|c|c|c|}
\hline \multirow{3}{*}{ ESPECIE } & \multicolumn{2}{|c|}{ ABUNDANCIA } & \multicolumn{2}{|c|}{ DOMINANCIA } & \multicolumn{2}{|c|}{ FRECUENCIA } & \multirow[t]{3}{*}{ IVI } \\
\hline & ABSOLUTA & RELATIVA & ABSOLUTA & RELATIVA & ABSOLUTA & RELATIVA & \\
\hline & $\left(\mathrm{n} \mathrm{ha}^{-1}\right)$ & $(\%)$ & $\left(\mathrm{m}^{2} \mathrm{ha}^{-1}\right)$ & $(\%)$ & & $(\%)$ & \\
\hline Acacia rigidula & 266 & 8,03 & 1750,59 & 10,50 & 64,29 & 5,60 & 8,04 \\
\hline Leucophyllum frutescens & 243 & 7,33 & 1211,61 & 7,27 & 80,00 & 6,97 & 7,19 \\
\hline Helietta parvifolia & 127 & 3,83 & 2178,88 & 13,07 & 37,14 & 3,24 & 6,71 \\
\hline Lippia graveolens & 462 & 13,95 & 245,31 & 1,47 & 44,29 & 3,86 & 6,43 \\
\hline Zanthoxylum fagara & 132 & 3,98 & 1449,67 & 8,70 & 44,29 & 3,86 & 5,51 \\
\hline Cordia boissieri & 86 & 2,60 & 1400,29 & 8,40 & 57,14 & 4,98 & 5,33 \\
\hline Chromolaena odorata & 314 & 9,48 & 788,38 & 4,73 & 4,29 & 0,37 & 4,86 \\
\hline Eysenhardtia texana & 79 & 2,38 & 788,38 & 4,73 & 61,43 & 5,35 & 4,16 \\
\hline Celtis pallida & 60 & 1,81 & 966,66 & 5,80 & 45,71 & 3,99 & 3,86 \\
\hline Havardia pallens & 85 & 2,57 & 915,52 & 5,49 & 37,14 & 3,24 & 3,77 \\
\hline Rhamnus humboldtiana & 142 & 4,29 & 129,94 & 0,78 & 54,29 & 4,73 & 3,27 \\
\hline Acacia greggii & 77 & 2,32 & 654,61 & 3,93 & 34,29 & 2,99 & 3,08 \\
\hline Diospyros texana & 36 & 1,09 & 824,56 & 4,95 & 27,14 & 2,37 & 2,80 \\
\hline Forestiera angustifolia & 52 & 1,57 & 483,08 & 2,90 & 34,29 & 2,99 & 2,49 \\
\hline Sideroxylon celastrinum & 37 & 1,12 & 445,37 & 2,67 & 34,29 & 2,99 & 2,26 \\
\hline Neopringlea integrifolia & 37 & 1,12 & 379,22 & 2,27 & 27,14 & 2,37 & 1,92 \\
\hline Agave lechuguilla & 76 & 2,29 & 69,84 & 0,42 & 32,86 & 2,86 & 1,86 \\
\hline Porlieria angustifolia & 69 & 2,08 & 54,20 & 0,33 & 32,86 & 2,86 & 1,76 \\
\hline Gymnosperma glutinosum & 149 & 4,50 & 70,24 & 0,42 & 2,86 & 0,25 & 1,72 \\
\hline Yucca filifera & 17 & 0,51 & 542,76 & 3,26 & 14,29 & 1,25 & 1,67 \\
\hline Dodonaea viscosa & 57 & 1,72 & 267,76 & 1,61 & 18,57 & 1,62 & 1,65 \\
\hline Castela tortuosa & 23 & 0,69 & 166,16 & 1,00 & 30,00 & 2,62 & 1,44 \\
\hline Jatropha dioica & 57 & 1,72 & 25,10 & 0,15 & 27,14 & 2,37 & 1,41 \\
\hline Colubrina texensis & 75 & 2,26 & 86,32 & 0,52 & 8,57 & 0,75 & 1,18 \\
\hline Agave asperrima & 40 & 1,21 & 12,13 & 0,07 & 25,71 & 2,24 & 1,17 \\
\hline Cylindropuntia leptocaulis & 31 & 0,94 & 6,82 & 0,04 & 27,14 & 2,37 & 1,11 \\
\hline Hechtia glomerata & 32 & 0,97 & 23,74 & 0,14 & 22,86 & 1,99 & 1,03 \\
\hline Parthenium incanum & 62 & 1,87 & 17,07 & 0,10 & 12,86 & 1,12 & 1,03 \\
\hline Opuntia engelmannii & 26 & 0,78 & 7,73 & 0,05 & 20,00 & 1,74 & 0,86 \\
\hline Croton torreyanus & 29 & 0,88 & 56,71 & 0,34 & 12,86 & 1,12 & 0,78 \\
\hline Turnera diffusa & 56 & 1,69 & 13,29 & 0,08 & 4,29 & 0,37 & 0,71 \\
\hline Acacia farnesiana & 13 & 0,39 & 157,71 & 0,95 & 8,57 & 0,75 & 0,70 \\
\hline Cercidium macrum & 11 & 0,33 & 79,13 & 0,47 & 12,86 & 1,12 & 0,64 \\
\hline Krameria ramosissima & 47 & 1,42 & 23,29 & 0,14 & 2,86 & 0,25 & 0,60 \\
\hline Ferocactus hamatacanthus & 18 & 0,54 & 0,62 & 0,00 & 12,86 & 1,12 & 0,56 \\
\hline Leucaena pulvurenta & 15 & 0,45 & 89,30 & 0,54 & 4,29 & 0,37 & 0,45 \\
\hline Ziziphus obtusifolia & 12 & 0,36 & 27,89 & 0,17 & 8,57 & 0,75 & 0,43 \\
\hline Ricinus communis & 26 & 0,78 & 41,99 & 0,25 & 1,43 & 0,12 & 0,39 \\
\hline Turbinicarpus saueri & 12 & 0,36 & 0,15 & 0,00 & 8,57 & 0,75 & 0,37 \\
\hline Coryphantha salinensis & 8 & 0,24 & 0,06 & 0,00 & 8,57 & 0,75 & 0,33 \\
\hline
\end{tabular}


Gayana Bot. 75(1), 2018

\begin{tabular}{|c|c|c|c|c|c|c|c|}
\hline \multirow{3}{*}{ ESPECIE } & \multicolumn{2}{|c|}{ ABUNDANCIA } & \multicolumn{2}{|c|}{ DOMINANCIA } & \multicolumn{2}{|c|}{ FRECUENCIA } & \multirow[t]{3}{*}{ IVI } \\
\hline & ABSOLUTA & RELATIVA & ABSOLUTA & RELATIVA & ABSOLUTA & RELATIVA & \\
\hline & $\left(\mathrm{n} \mathrm{ha}^{-1}\right)$ & $(\%)$ & $\left(\mathrm{m}^{2} \mathrm{ha}^{-1}\right)$ & $(\%)$ & & $(\%)$ & \\
\hline Echinocereus pentalophus & 8 & 0,24 & 0,03 & 0,00 & 8,57 & 0,75 & 0,33 \\
\hline Acacia wrightii & 9 & 0,27 & 55,58 & 0,33 & 4,29 & 0,37 & 0,33 \\
\hline Coryphantha nickelsie & 7 & 0,21 & 0,01 & 0,00 & 8,57 & 0,75 & 0,32 \\
\hline Corynopuntia schottii & 9 & 0,27 & 0,02 & 0,00 & 7,14 & 0,62 & 0,30 \\
\hline Ariocarpus retusus & 7 & 0,21 & 0,01 & 0,00 & 7,14 & 0,62 & 0,28 \\
\hline Neomammillaria candida & 7 & 0,21 & 0,01 & 0,00 & 7,14 & 0,62 & 0,28 \\
\hline Nicotiana glauca & 4 & 0,12 & 35,17 & 0,21 & 4,29 & 0,37 & 0,24 \\
\hline Ebenopsis ebano & 3 & 0,09 & 28,06 & 0,17 & 4,29 & 0,37 & 0,21 \\
\hline Prosopis glandulosa & 3 & 0,09 & 26,07 & 0,16 & 4,29 & 0,37 & 0,21 \\
\hline Schaefferia cuneifolia & 5 & 0,15 & 6,54 & 0,04 & 4,29 & 0,37 & 0,19 \\
\hline Bernardia myricifolia & 4 & 0,12 & 10,09 & 0,06 & 4,29 & 0,37 & 0,18 \\
\hline Lycium berlandieri & 4 & 0,12 & 9,26 & 0,06 & 4,29 & 0,37 & 0,18 \\
\hline Aloysia gratissima & 9 & 0,27 & 1,44 & 0,01 & 2,86 & 0,25 & 0,18 \\
\hline Condalia hookeri & 4 & 0,12 & 18,81 & 0,11 & 2,86 & 0,25 & 0,16 \\
\hline Echinocereus parkeri & 6 & 0,18 & 0,19 & 0,00 & 2,86 & 0,25 & 0,14 \\
\hline Colubrina greggii & 5 & 0,15 & 3,97 & 0,02 & 2,86 & 0,25 & 0,14 \\
\hline Randia laetevirens & 2 & 0,06 & 3,07 & 0,02 & 2,86 & 0,25 & 0,11 \\
\hline Echinocactus horizonthalonius & 2 & 0,06 & 0,10 & 0,00 & 2,86 & 0,25 & 0,10 \\
\hline Echinocactus texensis & 6 & 0,18 & 0,48 & 0,00 & 1,43 & 0,12 & 0,10 \\
\hline Parkinsonia acuelata & 1 & 0,03 & 13,74 & 0,08 & 1,43 & 0,12 & 0,08 \\
\hline Mammillaria prolifera & 3 & 0,09 & 0,12 & 0,00 & 1,43 & 0,12 & 0,07 \\
\hline Sclerocactus scheeri & 3 & 0,09 & 0,05 & 0,00 & 1,43 & 0,12 & 0,07 \\
\hline Ehretia anacua & 1 & 0,03 & 5,43 & 0,03 & 1,43 & 0,12 & 0,06 \\
\hline Echinocereus poselgeri & 2 & 0,06 & 0,05 & 0,00 & 1,43 & 0,12 & 0,06 \\
\hline Lantana achyranthifolia & 1 & 0,03 & 0,55 & 0,00 & 1,43 & 0,12 & 0,05 \\
\hline Lantana camara & 1 & 0,03 & 0,18 & 0,00 & 1,43 & 0,12 & 0,05 \\
\hline \multirow[t]{2}{*}{ Mammillaria heyderi } & 1 & 0,03 & 0,01 & 0,00 & 1,43 & 0,12 & 0,05 \\
\hline & 3313 & 100 & 16671,06 & 100 & 1147,14286 & 100 & 100 \\
\hline
\end{tabular}

TABla 4, Ajuste de los modelos a la distribución de la abundancia de especies en el área de estudio, Donde loglik= Logaritmo de verosimilitud; $\mathrm{AIC}=$ Criterio de Información de Akaike; $\mathrm{dAIC}=$ Delta, / Adjustment the models to the distribution of species abundance in the study area, Where loglik = log likelihood; AIC = Information Criterion of Akaike; dAIC =Delta,

\begin{tabular}{lccr}
\hline Modelo & loglik & AIC & dAIC \\
\hline Poisson Lognormal & $-314,10$ & 632,19 & 0,00 \\
Modelo Neutral & $-316,97$ & 635,95 & 3,76 \\
Modelo Geométrico & $-327,68$ & 657,36 & 25,17 \\
\hline
\end{tabular}




\section{DISCUSIÓN}

De acuerdo a los resultados obtenidos, se observa que la riqueza de especies arbóreas y arbustivas registrada en el matorral espinoso tamaulipeco del área de estudio (47) es mayor a la registrada por Mora et al. (2013) quienes reportaron 21 especies y menor a la registrada por Domínguez et al. (2013) quienes registraron 53 especies al evaluar la diversidad del MET en otras áreas maduras y sin perturbación utilizando el mismo método de muestreo. Sin embargo en estos estudios no se consideraron las especies suculentas, lo que hace que en el presente estudio se registre mayor riqueza (67 especies), dando muestra de la importancia de incluir las especies suculentas en este tipo de estudios. La familia Fabaceae y el género Acacia fueron los más representativos, coincidiendo con lo reportado en los estudios de composición y diversidad del matorral espinoso tamaulipeco de Mora et al. (2013) y Domínguez et al. (2013).

Comparando los valores obtenidos de los índices de Margalef $\left(D_{\mathrm{Mg}}=8,14\right)$ y Shannon $\left(H^{\prime}=3,36\right)$ con estudios realizados (Alanís 2006, Jiménez et al. 2012, Pequeño et al. 2012) en otras asociaciones vegetales de matorral que se desarrollan en el estado de Nuevo León, se puede deducir que se trata de un grupo funcional vegetal diverso. Estos resultados son similares a los reportados por otros autores (Canizales et al. 2009, Alanís et al. 2015a) que han evaluado asociaciones vegetales maduras del matorral submontano considerando especies arbóreas, arbustivas y suculentas utilizando el mismo método de muestreo. Alanís et al. (2015a) evaluaron un matorral submontano contiguo al Área Metropolitana de Monterrey (Noreste de México) y registraron valores de índice de Margalef $\left(D_{M g}=6,02\right)$ y Shannon $\left(H^{\prime}=3,02\right)$. También son equiparables con los resultados de Canizales et al. (2009) quienes caracterizaron un área del matorral submontano de la Sierra Madre Oriental, y reportaron valores de índice de Margalef $\left(D_{M g}=6,34\right)$ y Shannon $\left(H^{\prime}=3,00\right)$.

La abundancia registrada (3.313 ind $\mathrm{ha}^{-1}$ ) es superior a lo reportado por González et al. (2010) y Jiménez et al. (2012), los cuales registraron $1.741 \mathrm{n} \mathrm{ha}^{-1}$ y $2.370 \mathrm{n} \mathrm{ha}^{-1}$ respectivamente al evaluar un área del MET. Sin embargo, Alanís et al. (2015a) registraron una abundancia de 3.629 $\mathrm{n} \mathrm{ha} \mathrm{h}^{-1}$ al evaluar un matorral submontano contiguo al Área Metropolitana de Monterrey, considerando plantas arbóreas, arbustivas y suculentas. El valor de la cobertura de copas registrado $\left(16.671 \mathrm{~m}^{2} \mathrm{ha}^{-1}\right)$ indica que existe una cobertura superior al $100 \%$ y por lo tanto un considerable traslape de copa. El traslape de copa es continuamente registrado en estudios realizados en asociaciones vegetales maduras del matorral espinoso tamaulipeco, en donde no influye si se toman en consideración las especies suculentas (García \& Jurado 2008, Mora et al. 2013, Domínguez et al. 2013).

Las especies, Acacia rigidula, Leucophyllum frutescens y Helietta parvifolia fueron las que presentaron el mayor índice de valor de importancia. Estos resultados coinciden con lo reportado por González et al. (2010), quienes registraron a Acacia rigidula como la especie con mayor valor de importancia al evaluar la composición y estructura de la vegetación en tres sitios de Nuevo León. De la misma forma, Estrada et al. (2004) señalaron que es palpable el predominio de Acacia rigidula en el matorral espinoso tamaulipeco y matorral submontano. García \& Jurado (2008) reportaron a las especies Helietta parvifolia y Acacia rigidula como las especies con mayor valor de importancia en un estudio de caracterización del matorral con condiciones prístinas.

En la Tabla 3 se aprecia que las especies Lippia graveolens, Eupatorium odoratum, Acacia rigidula y Leucophyllum frutescens tienen una abundancia alta, mientras que Parkinsonia aculeata, Ehretia anacua, Lantana achyranthifolia, Lantana camara y Mammillaria heyderi son especies raras. Alanís-Rodríguez et al. (2015b) registraron a Acacia amentacea y Leucophyllum frutescens como especies con una abundancia alta al evaluar la estructura y diversidad del matorral submontano contiguo a la ciudad de Monterrey.

Las especies registradas en el área de estudio presentan una distribución de tipo log normal. Coincidiendo con Alanís et al. (2015a) quienes determinaron que las especies de un área del matorral submontano contiguo al área metropolitana de Monterrey, presentan una distribución de este mismo tipo. Generalmente la distribución de tipo Poisson-lognormal es registrada en estudios donde las asociaciones vegetales se encuentran en estado maduro (Long et al. 2012, Martella et al. 2012, Matthews \& Whittaker 2014, Alanís-Rodríguez et al. 2015b). Otros estudios señalan que este tipo de distribución es la más común en asociaciones vegetales sin disturbio (Zacarías-Eslava et al. 2011, Long et al. 2012, Martella et al. 2012, Matthews \& Whittaker 2014).

\section{CONCLUSIONES}

El área estudiada del MET presenta una alta riqueza específica y diversidad de especies arbóreas, arbustivas y suculentas en comparación con otras asociaciones vegetales del matorral del noreste de México. Las familias con mayor importancia por su contribución al grupo funcional vegetal estudiado son Fabaceae, Rutaceae, Asteraceae, Scrophulariaceae y Verbenaceae. Las especies más importantes son Acacia rigidula, Leucophyllum frutescens, Helietta parvifolia y Lippia graveolens. La curva de rango/ abundancia de las especies se ajustó mejor a una función log-normal, la cual es una distribución característica en la mayoría de las asociaciones vegetales maduras y sin perturbación. 


\section{AGRADECIMIENTOS}

Al Consejo Nacional de Ciencia y Tecnología por la beca de manutención otorgada al primer autor y a la Facultad de Ciencias Forestales de la Universidad Autónoma de Nuevo León por las facilidades otorgadas en la ejecución de la investigación.

\section{LITERATURA CITADA}

Alanís, E. 2006. Diversidad de especies arbóreas y arbustivas en áreas con distinto historial de uso antropogénico en el matorral espinoso tamaulipeco. Tesis de Maestría, Facultad de Ciencias Forestales, Universidad Autónoma de Nuevo León, Linares, N.L., México. 110 pp.

Alanís, E., Jiménez, J., Aguirre, O.A., Treviño, E.J., Jurado, E., González, M.A. 2008. Efecto del uso del suelo en la fitodiversidad del matorral espinoso tamaulipeco. Ciencia UANL 11: 56-62.

Alanís, E., Jiménez, J., Canizales, P.A., González, H., Mora-Olivo, A. 2015a. Estado actual del conocimiento de la estructura arbórea y arbustiva del matorral espinoso tamaulipeco del noreste de México. Revista Iberoamericana de Ciencias 2(7): 69-80.

Alanís, E., Jiménez, J., GonzÁlez, M.A., Yerena, J.I., Cuellar, L.G., MorA, A. 2013. Análisis de la vegetación secundaria del matorral espinoso tamaulipeco, México. Phyton International Journal of Experimental Botany 82: 185-192.

Alanís, F.G., Cano, C.G., Rovalo, M.M. 1996. Vegetación y flora de Nuevo León, una guía botánico ecológica. CEMEXImpresora Monterrey, Monterrey, N. L. México. 251 pp.

Alanís-Rodríguez, E., Jiménez-Pérez, J., Mora-Olivo, A., Martínez-Ávalos, J.G., Mata-Balderas, J.M., ChávezCosta, A.C., Rubio-CAmacho, E.A. 2015b. Estructura y diversidad del matorral submontano contiguo al área metropolitana de Monterrey, Nuevo León, México. Acta Botánica Mexicana 113: 1-19.

Canizales-Velázquez, P.A., Alanís-Rodríguez, E., ArandaRamos, R., Mata-Balderas, J.M., Jiménez-Pérez, J., Alanís-Flores, G., Uvalle-Sauceda, J.I., Ruíz-Bautista, M.G. 2009. Caracterización estructural del matorral submontano de la Sierra Madre Oriental, Nuevo León, México. Revista Chapingo, Series Ciencias Forestales y del Ambiente 15: 115-120.

Codron, J.C.G. 2011. 5.2.1 Las plantas del desierto: adaptación y oportunismo. URL: http://ocw.unican.es/cienciassociales-y-juridicas/biogeografia/materiales/tema5/5.2.1-las-plantas-del-desierto-adaptacion-y Accedido: Marzo 20, 2016.

Domínguez, T.G., González, H., Ramírez, R.G., Estrada, A.E., Cantú, I., Gómez, M.V., Villarreal, J.Á., Socorro, M., Alanís, G. 2013. Diversidad estructural del matorral espinoso tamaulipeco durante las épocas seca y húmeda. Revista Mexicana de Ciencias Forestales 4(17): 106-123.

Estrada, E., Yen, C., Delgado, A., Villarreal, J.A. 2004. Leguminosas del centro del estado de Nuevo León, México. Anales del Instituto de Biología, Universidad Nacional Autónoma de México. Serie Botánica 75: 73-85.
Foroughbakhch, R., Reyes-Reyna, G., Alvarado-VÁzquez, M.A., Hernández-Piñero, J.L., Rocha, A. 2005. Use of quantitative methods to determine leaf biomass on 15 woody shrub species in northeastern Mexico. Forest Ecology and Management 216: 359-366.

García, J., Jurado, E. 2008. Caracterización del matorral con condiciones prístinas en Linares N.L., México. Ra Ximhai 4(1): 1-21.

González, H., Ramírez, R.G., Cantú, I., Gómez, M., Uvalle, J.I. 2010. Composición y estructura de la vegetación en tres sitios del estado de Nuevo León, México. Polibotánica 29: 91-106.

Heiseke, D., Foroughbakhch, R. 1985. El matorral como recurso forestal. Reporte científico No. 1. Facultad de Silvicultura y Manejo de Recursos Renovables. Universidad Autónoma de Nuevo León. 31 pp.

Hernández-Livera,A.E. 1996. Cactáceas mexicanas: Problemática, y perspectivas en el IREGEP. Boletín informativo del instituto de recursos genéticos y productividad. Colegio de postgraduados en ciencias agrícolas. De enero-abril, No. 3-4.

Jiménez, J., Alanís, E., Ruiz, J.L., GonzÁlez, M.A., Yerena, J.I., Alanís, G.J. 2012. Diversidad de la regeneración leñosa del matorral espinoso tamaulipeco con historial agrícola en el NE de México. Ciencia UANL 15: 66-71.

Jiménez, J., Alanís, E., González, M.A., Aguirre, O.A., Treviño, E.J. 2013. Characterizing woody species regeneration in areas with different land history tenure in the tamaulipan thornscrub, Mexico. The Southwestern Naturalist 58: 299304.

Long, W., YAng, X., Li, D. 2012. Patterns of species diversity and soil nutrients along a chronosequence of vegetation recovery in Hainan Island, South China. Ecological Research 27(3): 561-568.

MagurRan, A.E. 2004. Measuring biological diversity. Blackwell, Cambridge, Massachusetts, USA. 256 pp.

Martella, M., Trumper, E., Bellis, L.M., Renison, D., Giordano, P., Bazzano, G., Gleisser, R. 2012. Manual de ecología. Evaluación de la biodiversidad. Reduca (Biología) Serie Ecología 5(1): 71-115.

Matthews, T.J., Whittaker, R.J. 2014. Fitting and comparing competing models of the species abundance distribution: assessment and prospect. Frontiers of Biogeography. 6: 67-82.

Mora, C.A., Alanís, E., JimÉnez, J., GonZÁlez, M.A., Yerena, J.I., Cuellar, L.G. 2013. Estructura, composición florística y diversidad del matorral espinoso tamaulipeco, México. Ecología Aplicada 12(1): 29-34.

Moreno, C.E. 2001. Métodos para medir la biodiversidad. Manual y tesis SEA. Editado por Cooperación Iberoamericana (CYTED), Unesco (Orcyt) y SEA. Vol. 1. Pachuca, Hidalgo, México. 83 pp.

Mostacedo, B., Fredericksen, S. 2000. Manual de métodos básicos de muestreo y análisis en ecología vegetal. BOLFOR; Santa Cruz, Bolivia. 87 pp.

Müeller -Dombois, D., Ellenberg, H. 1974. Aims and methods of vegetation ecology. John Wiley and Sons, Nueva York. 547 pp.

Pequeño, L.M., Alanís, E., JimÉnez, J., GonzÁlez, M.A., Yerena, I., Cuellar, L.G., Mora, A. 2012. Análisis de la restauración 
Caracterización del matorral espinoso tamaulipeco: GraCIANO-ÁvILA, G. ET AL.

pasiva post-pecuaria en el matorral espinoso tamaulipeco del noreste de México. Ciencia UAT 24(2): 48-53.

Prado, P.I., Miranda, M.D., Chalom, A. 2014. Sads: Maximum Likelihood Models for Species Abundance Distributions. R package version 0.1, 10. URL: http://cran.r-project.org/ web/packages/sads/index.html Viewed: November 25, 2015.

R Development Core Team, R. 2011. R: A Language and Environment for Statistical Computing. (R.D.C. Team, Ed.) $R$ Foundation for Statistical Computing. R Foundation for Statistical Computing. doi:10.1007/978-3-540-746867.

Ramírez-Lozano, R., Domínguez-Gómez, T.G., GonzálezRodríguez, H., Cantú-Silva, I., Gómez Meza, M.V., Sarquís-Ramírez, J.I., Jurado, E. 2013. Composición y diversidad de la vegetación en cuatro sitios del noreste de México. Madera y Bosques 19: 59-72.

Reid, N., Smith, S., Beyer-Münzel, P., Marroquin, J. 1990. Floristic and structural variation in the Tamaulipan Thornscrub, Northeastern México. Journal of Vegetation Science 1: 529-538.

Rzedowski, J. 1978. Vegetación de México, Ed. Limusa, México. $432 \mathrm{pp}$.
Rzedowski, J. 2006. Vegetación de México. 1ra. Edición digital. Comisión Nacional para el Conocimiento y Uso de la Biodiversidad, México. 255 pp.

SÁnchez, J.G., Espinosa, A., GonzÁlez, M.A. 2010. La sociedad de cactáceas y suculentas de Nuevo León, A.C. Ciencia UANL, 13(3): 226-229.

SARH. 1994. Inventario Nacional Forestal Periódico, Memoria Nacional. México. Secretaría de Agricultura y Recursos Hidráulicos. Subsecretaría Forestal y de Fauna Silvestre. $74 \mathrm{pp}$.

SHAnNon, C.E. 1948. The mathematical theory of communication. In: Shannon, C.E., Weaver, W. (eds.), The mathematical theory of communication. University of Illinois Press. Urbana, IL, USA. pp. 29-125.

The Plant List. 2013. Version 1.1. URL: http://www.theplantlist. org/ Viewed: March 10, 2016.

Whittaker, R.H. 1972. Evolution and measurement of species diversity. Taxon 21: 213-251.

Zacarías-Eslava, L.E., Cornejo-Tenorio, G., Cortés-Flores, J., González-Castañeda, N., Ibarra-Manríquez, G. 2011. Composición, estructura y diversidad del cerro El Águila, Michoacán, México. Revista Mexicana de Bodiversidad 82(3): 854-869.

Recibido: 18.12 .2015

Aceptado: 10.11 .2017 